\title{
Existence of standing waves for quasi-linear Schrödinger equations on $\mathrm{T}^{n}$
}

https://doi.org/10.1515/anona-2020-0038

Received June 7, 2019; accepted June 14, 2019.

Abstract: This paper is devoted to the study of the existence of standing waves for a class of quasi-linear Schrödinger equations on $\mathbf{T}^{n}$ with dimension $n \geq 3$. By construction of a suitable Nash-Moser-type iteration scheme, we overcome the clusters of "small divisor" problem, then the existence of standing waves for quasilinear Schrödinger equations is established.

Keywords: Schrödinger equation; Small divisors; Periodic solution

MSC: 35J60; 35R03; 35B10

\section{Introduction and Main Results}

This paper considers the quasi-linear Schrödinger equation

$$
i U_{t}-\triangle U-a\left(\nabla^{q} U\right)=f(x,|U|) U, \quad(t, x) \in \mathbf{R} \times \mathbf{T}^{n},
$$

where $U$ is a complex-valued functions of $(t, x), \mathbf{T}^{n}$ is a $n$-dimensional flat torus with $n \geq 3, \triangle$ is the LaplaceBeltrami operator, $q \geq 1$, the terms $a(s)$ and $f(x, s)$ satisfy gauge invariant, i.e. $a\left(e^{i \varphi} s\right)=e^{i \varphi} a(s)$ for almost every $x \in \mathbf{T}^{n}$, all $\varphi \in \mathbf{R}$ and $s \geq 0$.

The problem in this general setting arises in various fields of mathematical physics, such as the superfluid film equation of fluid mechanics [16] and ferromagnets and magnons [2, 15]. Lange etc [17] has obtained the local existence and uniqueness of smooth solution for a class of quasilinear Schrödinger equation. Poppenberg [22] used the Nash-Moser implicit function theorem to overcome "the loss of derivatives" introduced by the nonlinearity. Kenig etc [14] studied the Cauchy problem of a more general class of quasilinear Schrödinger equation. Bahrouni-Ounaies-Rădulescu [1] studied compactly supported solutions of Schrödinger equations with small perturbation. Zhang-Zhang-Xiang [27] obtained the existence of ground states for fractional Schrödinger equations involving a critical nonlinearity. Xue-Tang [25] showed that the existence of a bound state solution for quasilinear Schrödinger equations. One can see [8, 9, 12, 21] for more results on the existence of solution for elliptic equations in $\mathbf{R}^{n}$. To our knowledge, there is no result on the existence of standing waves for a class quasilinear Schrödinger equation with higher derivatives in higher dimension flat-torus $\mathbf{T}^{n}$.

A standing wave is a solution of the form

$$
U(t, x)=e^{i \mu t} u(x), \mu>0,
$$

and for solutions of this form, quasi-linear Schrödinger equation (1.1) is reduced into a quasi-linear elliptic equation involving the parameter $\mu$

$$
-\triangle u-\mu u-a\left(\nabla^{q} u\right)=f(x,|u|) u .
$$

\footnotetext{
Xin Zhao, School of Mathematics and Big Data, Huizhou University, Huizhou, P.R. China

*Corresponding Author: Weiping Yan, School of Mathematics, Xiamen University, Xiamen, P.R. China, E-mail: yanwp@xmu.edu.cn
} 
We make assumptions on nonlinear terms $f$, which include the standard tame estimates and Taylor tame estimates. $f \in \mathbf{C}^{\infty}\left(\mathbf{T}^{n} \times \mathbf{R}, \mathbf{R}\right), f(0,0)=0, \partial_{u} f(x, 0)=\cdots=\left(\partial_{u}^{p-1}\right) f(x, 0)=0, \partial_{u}^{p} f(x, 0) \neq 0,1 \leq p \leq k, k \geq 2$ and

$$
\begin{gathered}
\left\|\partial_{u} f\left(x, u^{\prime}\right) u\right\|_{s} \leq c(s)\left(\|u\|_{s}^{p-1}+\left\|u^{\prime}\right\|_{s}\|u\|_{s_{0}}^{p-1}\right), \\
\left\|f\left(x, u+u^{\prime}\right)-f\left(x, u^{\prime}\right)-D_{u} f\left(x, u^{\prime}\right) u\right\|_{s} \leq c(s)\left(\left\|u^{\prime}\right\|_{s}\|u\|_{s_{0}}^{p-1}+\|u\|_{s_{0}}\|u\|_{s}^{p-1}\right),
\end{gathered}
$$

where $s>s_{0}>0, p>1, \forall u, u^{\prime} \in \mathbf{H}_{s}$ such that $\|u\|_{s_{0}} \leq 1$ and $\left\|u^{\prime}\right\|_{s_{0}} \leq 1$. In particular, for $s_{0}=s$,

$$
\left\|f\left(x, u+u^{\prime}\right)-f\left(x, u^{\prime}\right)-D_{u} f\left(x, u^{\prime}\right) u\right\|_{s} \leq c(s)\|u\|_{s}^{p} .
$$

In fact, when $p=2$, assumption (1.3) and (1.4) are natural for $f \in \mathbf{C}^{\infty}\left(\mathbf{T}^{n} \times \mathbf{R}, \mathbf{R}\right)$, which are tame estimates and Taylor tame estimates, respectively.

Rescaling in (1.2) amplitude $u(x) \mapsto \delta u(x), \delta>0$, we solve the following problem

$$
-\triangle u-\mu u-\varepsilon a\left(\nabla^{q} u\right)=\varepsilon f(\delta, u)
$$

where $a(s):=a s^{q}, f(\delta, u):=b(x) s^{p}+O(\delta), 1 \leq p \leq k$ and $\varepsilon=\delta^{p-1}$.

The problem of solving nonlinear elliptic equations with a singular perturbation was inspired by the work of Rabinowitz [24]. By employing the Nash-Moser iteration process, he proved that the elliptic singular perturbation problem has a uniqueness spatial periodic solution. For more related work, we refer to $[13,20]$. Han-Hong-Lin [10] partially extended the work of Rabinowitz [24], they considered the following singular perturbation problem

$$
-\triangle u+u+\varepsilon a\left(\nabla^{q} u\right)=f(x), \quad x \in \mathbf{R}^{2},
$$

where $q \geq 4$, the function $a(x)$ is smooth and $f(x)$ is $(2 \pi)^{2}$-periodic. Under some assumptions on $a(x)$ and $f(x)$, they employed the Nash-Moser iteration process to prove that above singular problem had spatial periodic solutions. But they only dealed with small divisors problem in one dimensional case. Beacuse there is the "clusters of small divisors" problem in higher dimensional case. The aim of the present paper is to focus on the solution of the small divisors problem in presence of large clusters and with smooth nonlinearities for singular perturbation elliptic problem (1.5) in higher dimensional case $(n \geq 3)$.

We will divide into two cases to discuss the existence of solutions for (1.5). The first case is $a(x)=a x$, where $a \neq 0$ is a constant, then the "small divisor" phenomenon appears. The second case is $a(\cdot) \in \mathbf{C}^{\infty}(\mathbf{R})$. The second case is simpler than the first case, and we can use the Nash-Moser iteration scheme constructed in the first case to solve it. In what follows, we deal with the first case, i.e. $a\left((-1)^{q} \triangle^{q} u\right)=(-1)^{q} a \triangle^{q} u$. Thus we can rewrite (1.5) as

$$
-\triangle u-\mu u-(-1)^{q} \varepsilon a \triangle^{q} u=\varepsilon f(\delta, u) .
$$

Assume that $a$ is an irrational number and diophantine, i.e. there are constants $y_{0}>0, \tau_{0}>1$, such that

$$
|m-a n| \geq \frac{y_{0}}{|n|^{\tau_{0}}}, \quad \forall(m, n) \in \mathbf{Z}^{2} \backslash\{(0,0)\} .
$$

Then there exist $y>0$ and $\tau>1$ such that the first order Melnikov nonreonance condition

$$
\left|\omega_{j}^{2}-\mu-\varepsilon a \omega_{j}^{2 q}\right| \geq \frac{y}{|j|^{\tau}},
$$

where $\omega_{j}^{2} ;=|j|^{2}$ and $j \in \mathbf{Z}^{n}$.

Our main results are based on the Nash-Moser iterative scheme, which is firstly introduced by Nash [19] and Moser [18]. One can also see[11] for more details. Berti and Procesi [4] developed suitable linear and nonlinear harmonic analysis on compact Lie groups and homogeneous spaces, and via the technique and the Nash-Moser implicit function theorem, they found a family of time-periodic solutions of nonlinear 
Schrödinger equations and wave equations. Inspired by the work of $[4,5,26]$, we will construct a suitable Nash-Moser iteration scheme to study the elliptic-type singular perturbation problems (1.2) in higher dimensional flat torus.

We define the Sobolev scale of Hilbert spaces

$$
\mathbf{H}_{s}:=\mathbf{H}_{s}\left(\mathbf{T}^{n}, \mathbf{C}\right)=\left\{u(x)=\sum_{j \in \mathbf{T}^{n}} e^{i j \cdot x} u_{j}, u_{j}^{*}=\left.u_{-j}\left|\|u\|_{s}^{2}:=\sum_{j \in \mathbf{T}^{n}} e^{2|j| s}\right| u_{j}\right|^{2}<+\infty\right\}
$$

for some $s>\frac{n+1}{2}$. There holds $\|u v\|_{s} \leq\|u\|_{s}\|v\|_{s}$.

For the case $a(x)=a x$ in (1.2), we have the following result.

Theorem 1.1. Assume that $a>0$ is diophantine. For $\delta_{0}>0, s_{0}, k \in \boldsymbol{N}$ and $f \in \boldsymbol{C}^{\infty}$ satisfying (1.3)-(1.4), Then there exists a positive measure Cantor set $\mathcal{C} \subset\left[0, \delta_{0}\right]$ such that, $\forall a \in \mathcal{C}, U(t, x)=e^{i \mu t} u_{\delta}(x, \varepsilon)$ is a unique standing wave solution of (1.1). Furthermore, there exists a curve

$$
u(x, \varepsilon) \in \boldsymbol{C}^{1}\left(\left[0, \delta_{0}\right] ; \boldsymbol{H}_{s_{0}}\right) \text { with }\|u(\delta)\|_{s_{0}}=O(\delta) .
$$

For the second case, we consider equation (1.5) and assume that $a \in \mathbf{C}^{\infty}(\mathbf{R}), a(0)=0$, and

$$
\begin{gathered}
\left\|\partial_{u} a\left(u^{\prime}\right) u\right\|_{s} \leq c(s)\left(\|u\|_{s}^{p-1}+\left\|u^{\prime}\right\|_{s}\|u\|_{s_{0}}^{p-1}\right), \\
\left\|a\left(u+u^{\prime}\right)-a\left(u^{\prime}\right)-D_{u} a\left(u^{\prime}\right) u\right\|_{s} \leq c(s)\left(\left\|u^{\prime}\right\|_{s}\|u\|_{s_{0}}^{p-1}+\|u\|_{s_{0}}\|u\|_{s}^{p-1}\right),
\end{gathered}
$$

where $s>s_{0}>0,1<p \leq k, \forall u, u^{\prime} \in \mathbf{H}_{s}$ such that $\|u\|_{s_{0}} \leq 1$ and $\left\|u^{\prime}\right\|_{s_{0}} \leq 1$. In particular, for $s_{0}=s$,

$$
\left\|a\left(u+u^{\prime}\right)-a(u)-D_{u} a(u) u\right\|_{s} \leq c(s)\|u\|_{s}^{p} .
$$

For the second case, we have

Theorem 1.2. There exist $s_{0}$ and $k \in \boldsymbol{N}$ such that $\forall f, a \in \boldsymbol{C}^{\infty}$ satisfying (1.3)-(1.4) and (1.9)-(1.10), respectively. Then equation (1.1) admits a unique standing wave solution $U(t, x)=e^{i \mu t} u(x)$ with $u(x) \in \boldsymbol{H}_{s_{0}}$.

The proof of Theorem 1.2 is similar to the proof of Theorem 1.1, hence we omit it.

The structure of the paper is as follows: In next section, we show that the linearized equation of (1.6) is solvable by means of proving the invertible of its linearized operator. Section 3 gives the proof of Theorem 1.1 by construction of a suitable Nash-Moser iteration scheme.

\section{Analysis of the Linearized operator}

This section is devoted to prove the invertible of linearized operator

$$
\mathbf{L}:=-\triangle-\mu-(-1)^{q} \varepsilon a \triangle^{q}-\varepsilon \partial_{u} f(\delta, u) .
$$

We define the finite dimensional subspace of $\mathbf{H}_{s}$ as

$$
\mathbf{H}^{A}:=\operatorname{Span}_{j \in A} e_{j}=\left\{\sum_{k \in A} h_{j} e_{j}: h_{j} \in \mathbf{C}, h_{j}^{\star}=h_{-j}\right\},
$$

where $A$ is a finite and symmetric subset of $\mathbf{Z}^{n+1}$ and $e_{j}(x)=e^{i j \cdot x}$.

For $\forall h=\sum_{j \in \mathbf{Z}^{n}} h_{j} e_{j} \in \mathbf{H}_{s}$, We denote

$$
P_{A} h=\sum_{j \in A} h_{j} e_{j},
$$

which is a $\mathbf{L}^{2}$-orthogonal projector on $\mathbf{H}^{A}$. 
Let $A=\Omega_{N}:=\left\{j \in \mathbf{Z}^{n}|| j \mid \leq N\right\}$ and $b(x):=-\left(\partial_{u} f\right)(\delta, u)$. Then the operator (2.1) can be defined on $\mathbf{H}^{\Omega_{N}}:=\mathbf{H}^{(N)}$, i.e.

$$
h \mapsto \mathbf{L}^{(N)}[h]:=L_{a} h+\varepsilon P_{\Omega_{N}}(b(x) h), \quad \forall h \in \mathbf{H}^{(N)},
$$

where $L_{a}:=-\triangle-m-(-1)^{q} \varepsilon a \triangle^{q}$.

We write the linearized operator in (2.2) by the block matrix

$$
\mathbf{L}^{(N)}=D+\varepsilon T, \quad D:=L_{a} .
$$

In the $L^{2}$-orthonormal basis $\left(e_{j}\right)_{j \in \Omega_{N}}$ of $\mathbf{H}^{\Omega_{N}}, D$ is represented a diagonal matrix with eigenvalues

$$
D_{j}:=|j|^{2}-m-\varepsilon a|j|^{2 q},
$$

whereas $T$ is represented by the self-adjoint Toepliz matrix $\left(b_{j-j^{\prime}}\right)_{j, j^{\prime} \in \Omega_{N}}$, the $b_{j}$ is the Fourier coefficients of the function $b(x)$.

Now we give the main result in this section.

Proposition 2.1. Assume that

$$
|m-a n| \geq \frac{y_{1}}{\max \left(1,|m|^{\frac{3}{2}}\right)}, 0<y_{1}<1, \forall(m, n) \in \boldsymbol{Z}^{2} \backslash\{(0,0)\},
$$

and $\|u\|_{\bar{\sigma}} \leq 1, \forall 1 \leq r \leq N, \forall \kappa \geq 1$,

$$
\left\|\left(L_{a}^{(r)}(\delta, u)\right)^{-1}\right\|_{0} \leq \frac{4 r^{K}}{y_{1}} .
$$

Then the linearized operator $\boldsymbol{L}^{(N)}(\delta, u)$ is invertible and $\forall s_{2}>s_{1}>\bar{\sigma}>0$, the linearized operator $L_{a}^{(N)}$ satisfies

$$
\left\|\left(\boldsymbol{L}^{(N)}(\delta, u)\right)^{-1} h\right\|_{s_{1}} \leq C\left(s_{2}-s_{1}\right) N^{\tau+\kappa_{0}}\left(1+\varepsilon \varsigma^{-1}\|u\|_{S_{2}}^{p}\right)^{3}\|h\|_{s_{2}},
$$

where $C\left(s_{2}-s_{1}\right)=c\left(s_{2}-s_{1}\right)^{-\tau}$, c denotes a constant.

For fixing $\varsigma>0$, we define the regular sites $R$ and the singular sites $S$ as

$$
R:=\left\{j \in \Omega_{N}|| D_{j} \mid \geq \varsigma\right\} \text { and } S:=\left\{j \in \Omega_{N}|| D_{j} \mid<\varsigma\right\} .
$$

The following result shows the separation of singular sites, and the proof can be found in the paper [3, 4], so we omit it.

Lemma 2.1. Assume that $a$ is diophantine and a satisfies (2.5). There exists $\varsigma_{0}(y)$ such that for $\varsigma \in\left(0, \varsigma_{0}(y)\right]$ and a partition of the singular sites $S$ which can be partitioned in pairwise disjoint clusters $\Omega_{\alpha}$ as

$$
S=\bigcup_{\alpha \in A} \Omega_{\alpha}
$$

satisfying

- (dyadic) $\forall \alpha, M_{\alpha} \leq 2 m_{\alpha}$, where $M_{\alpha}:=\max _{j \in \Omega_{\alpha}}|j|, m_{\alpha}:=\max _{j \in \Omega_{\alpha}}|j|$.

- (separation) $\exists \lambda, c>0$ such that $d\left(\Omega_{\alpha}, \Omega_{\beta}\right) \geq c\left(M_{\alpha}+M_{\beta}\right)^{\lambda}, \forall \alpha \neq \beta$, where $d\left(\Omega_{\alpha}, \Omega_{\beta}\right):=\max _{j \in \Omega_{\alpha}, j^{\prime} \in \Omega_{\beta}} \mid j-$ $j^{\prime} \mid$

We define the polynomially localized block matrices

$$
\mathcal{A}_{s}:=\left\{A=\left(A_{j}^{j^{\prime}}\right)_{j, j^{\prime} \in \mathbf{Z}^{n}}:|A|_{s}^{2}:=\sup _{j \in \mathbf{Z}^{n}} \sum_{j^{\prime} \in \mathbf{Z}^{n}} e^{2 s\left|j-j^{\prime}\right|}\left\|A_{j}^{j^{\prime}}\right\|_{0}^{2}<\infty\right\},
$$

where $\left\|A_{j}^{j^{\prime}}\right\|_{0}:=\sup _{u \in \mathbf{H}^{(N)},\|u\|_{0}=1}\left\|A_{j}^{j^{\prime}} u\right\|_{0}$ is the $\mathbf{L}^{2}$-operator norm in $\mathcal{L}\left(\mathbf{H}^{(N)}, \mathbf{H}^{(N)}\right)$. If $s^{\prime}>s$, then these holds $\mathcal{A}_{s^{\prime}} \subset \mathcal{A}_{s}$.

The next lemma (see [4]) shows the algebra property of $\mathcal{A}_{s}$ and interpolation inequality. 
Lemma 2.2. There holds

$$
\begin{aligned}
& |A B|_{s} \leq c(s)|A|_{s}|B|_{s}, \quad \forall A, B \in \mathcal{A}_{s}, \quad s>s_{0}>\frac{r+n+1}{2}, \\
& |A B|_{s} \leq c(s)\left(|A|_{s}|B|_{s_{0}}+|A|_{s_{0}}|B|_{s}\right), \quad s \geq s_{0}, \\
& \|A u\|_{s} \leq c(s)\left(|A|_{s}\|u\|_{s_{0}}+|A|_{s_{0}}\|u\|_{s}\right), \quad \forall u \in \boldsymbol{H}_{s}, \quad s \geq s_{0} .
\end{aligned}
$$

By Lemma 2.2, we can get, $\forall m \in \mathbf{N}$,

$$
\begin{gathered}
\left|A^{m}\right|_{s} \leq c(s)^{m-1}|A|_{s}^{m}, \\
\left|A^{m}\right|_{s} \leq m\left(c(s)|A|_{s_{0}}\right)^{m-1}|A|_{s} .
\end{gathered}
$$

Then by the same method as the proof process of Lemma 6.3 in [4], we can prove the following result. Here we omit the proof.

Lemma 2.3. Let $s>s^{\prime}$. For a real $b \in \boldsymbol{H}_{s+s^{\prime}}$, the matrix $T=\left(T_{j}^{j^{\prime}}\right)_{j, j^{\prime} \in J_{N}^{+}}$defined in (2.3) is self-adjoint and belongs to the algebra of polynomially localized matrices $\mathcal{A}_{s}$ with

$$
|T|_{s} \leq K(s)\|b\|_{s+s^{\prime}}
$$

Moreover, for any $s>s^{\prime}$,

$$
|T|_{s} \leq K^{\prime}(s) N^{s^{\prime}}\|b\|_{s} .
$$

Since the decomposition

$$
\mathbf{H}^{(N)}:=\mathbf{H}_{R} \oplus \mathbf{H}_{S},
$$

we can represent the operator $\mathbf{L}^{(N)}$ as the self-adjoint block matrix

$$
\mathbf{L}^{(N)}=\left(\begin{array}{cc}
L_{R} & L_{R}^{S} \\
L_{S}^{R} & L_{S}
\end{array}\right),
$$

where $L_{R}^{S}=\left(L_{S}^{R}\right)^{\dagger}, L_{R}=L_{R}^{\dagger}, L_{S}=L_{S}^{\dagger}$.

Thus the invertibility of $\mathbf{L}^{(N)}$ can be expressed via the "resolvent-type" identity

$$
\left(\mathbf{L}^{(N)}\right)^{-1}=\left(\begin{array}{cc}
I & -L_{R}^{-1} L_{R}^{S} \\
0 & I
\end{array}\right)\left(\begin{array}{cc}
L_{R}^{-1} & 0 \\
0 & \mathcal{L}^{-1}
\end{array}\right)\left(\begin{array}{cc}
I & 0 \\
-L_{S}^{R} L_{R}^{-1} & I
\end{array}\right),
$$

where the "quasi-singular" matrix

$$
\mathcal{L}:=L_{S}-L_{S}^{R} L_{R}^{-1} L_{R}^{S} \in \mathcal{A}_{S}(S) .
$$

The reason of $\mathcal{L} \in \mathcal{A}_{S}(S)$ is that $\mathcal{L}$ is the restriction to $S$ of the polynomially localized matrix

$$
I_{S}\left(L-I_{S} L I_{R} \tilde{L}^{-1} I_{R} L I_{S}\right) I_{S} \in \mathcal{A}_{s},
$$

where

$$
\tilde{L}^{-1}=\left(\begin{array}{cc}
I & 0 \\
0 & L_{R}
\end{array}\right)
$$

Lemma 2.4. Assume that $a$ is diophantine. For $s_{0}<s_{1}<s_{2}<k-1,\left|L_{R}^{-1}\right| s_{0} \leq 2 \varsigma^{-1}$, the operator $L_{R}$ satisfies

$$
\begin{aligned}
& \left|\tilde{L}_{R}^{-1}\right|_{s_{1}} \leq c\left(s_{1}\right)\left(1+\varepsilon \varsigma^{-1}|T|_{s_{1}}\right), \\
& \left\|L_{R}^{-1} h\right\|_{s_{1}} \leq c\left(y, \tau, s_{2}\right)\left(s_{2}-s_{1}\right)^{-\tau}\left(1+\varepsilon \varsigma^{-1}|T|_{s_{2}}\right)\|h\|_{s_{2}},
\end{aligned}
$$

where $\tilde{L}^{-1}=L_{R}^{-1} D_{R}, c\left(y, \tau, s_{2}\right)$ is a constant depending on $y, \tau, s_{2}$. 
Proof. It follows from (2.3) and (2.8) that $D_{R}$ is a diagonal matrix and satisfies $\left|D_{R}^{-1}\right|_{s} \leq \varsigma^{-1}$. By (2.10), we have that the Neumann series

$$
\tilde{L}_{R}^{-1}=L_{R}^{-1} D_{R}=\sum_{m \geq 0}(-\varepsilon)^{m}\left(D_{R}^{-1} T_{R}\right)^{m}
$$

is totally convergent in $|\cdot| s_{1}$ with $\left|L_{R}^{-1}\right| s_{0} \leq 2 \varsigma^{-1}$, by taking $\varepsilon \varsigma^{-1}|T|_{s_{0}} \leq c\left(s_{0}\right)$ small enough.

Using (2.10) and (2.14), we have that $\forall m \in \mathbf{N}$,

$$
\begin{aligned}
\varepsilon^{m}\left|\left(D_{R}^{-1} T_{R}\right)^{m}\right|_{s_{1}} & \leq \varepsilon^{m} c(s)\left|\left(D_{R}^{-1} T_{R}\right)^{m}\right|_{s_{1}} \\
& \leq c(s) \varepsilon^{m} m\left(c(s)\left|D_{R}^{-1} T_{R}\right|_{s_{0}}\right)^{m-1}\left|D_{R}^{-1} T_{R}\right|_{s_{1}} \\
& \leq c^{\prime}(s) \varepsilon m \varsigma^{-1}\left(\varepsilon c\left(s_{1}\right) \varsigma^{-1}|T|_{s_{0}}\right)^{m-1}|T|_{s_{1}},
\end{aligned}
$$

which together with (2.18) implies that for $\varepsilon \varsigma^{-1}|T| s_{0}<c\left(s_{0}\right)$ small enough, (2.16) holds.

By non-resonance condition (1.8) and $\sup _{x>0}\left(x^{y} e^{-x}\right)=\left(y e^{-1}\right)^{y}, \forall y \geq 0$, we derive

$$
\begin{aligned}
e^{-2|j|\left(s_{2}-s_{1}\right)}\left|\omega_{j}^{2}+\mu-\varepsilon a \omega_{j}^{2 q}\right|^{-2} & \leq y^{-1}|j|^{\tau} e^{-2|j|\left(s_{2}-s_{1}\right)} \\
& \leq c(y, \tau)\left(s_{2}-s_{1}\right)^{-2 \tau} .
\end{aligned}
$$

Then by (2.19), for any $h \in \mathbf{H}_{R}$,

$$
\begin{aligned}
\left\|L_{R}^{-1} h\right\|_{s_{1}}^{2} & =\sum_{j \in R} e^{2|j| s_{1}}\left\|L_{R}^{-1} h_{j}\right\|_{\mathbf{L}^{2}}^{2} \\
& \leq \sum_{j \in R} e^{2|j| s_{1}}\left|\omega_{j}^{2}+\mu-\varepsilon a \omega_{j}^{2 q}\right|^{-2}\left\|\tilde{L}_{R}^{-1} h_{j}\right\|_{\mathbf{L}^{2}}^{2} \\
& \leq \sum_{j \in R} e^{-2|j|\left(s_{2}-s_{1}\right)}\left|\omega_{j}^{2}+\mu-\varepsilon a \omega_{j}^{2 q}\right|^{-2} e^{2|j| s_{2}}\left\|\tilde{L}_{R}^{-1} h_{j}\right\|_{\mathbf{L}^{2}}^{2} \\
& \leq c(y, \tau)\left(s_{2}-s_{1}\right)^{-2 \tau}\left\|\tilde{L}_{R}^{-1} h\right\|_{s_{2}}^{2} .
\end{aligned}
$$

Thus using interpolation (2.12) and (2.16), we derive that for $s_{1}<s<s_{2}$,

$$
\begin{aligned}
\left\|L_{R}^{-1} h\right\|_{s_{1}} & \leq c(y, \tau)\left(s_{2}-s_{1}\right)^{-\tau}\left\|\tilde{L}_{R}^{-1} h\right\|_{s_{2}} \\
& \leq c\left(r, \tau, s_{2}\right)\left(s_{2}-s_{1}\right)^{\tau}\left(\left|\tilde{L}_{R}^{-1}\right|_{s_{2}}\|h\|_{s}+\left|\tilde{L}_{R}^{-1}\right|_{s}\|h\|_{s_{2}}\right) \\
& \leq c\left(r, \tau, s_{2}\right)\left(s_{2}-s_{1}\right)^{\tau}\left(1+\varepsilon \varsigma^{-1}|T|_{s_{2}}\right)\|h\|_{s_{2}} .
\end{aligned}
$$

This completes the proof.

Next we analyse the quasi-singular matrix $\mathcal{L}$. By (2.9), the singular sites restricted to $J_{N}^{+}$are

$$
S=\bigcup_{\alpha \in l_{N}} \Omega_{\alpha}, \text { where } l_{N}:=\left\{\alpha \in \mathbf{N} \mid m_{\alpha} \leq N\right\} .
$$

Since the decomposition $\tilde{H}_{S}:=\bigoplus_{\alpha \in l_{N}} \tilde{H}_{\alpha}$, where $\mathbf{H}_{\alpha}:=\bigoplus_{j \in \Omega_{\alpha}} \mathcal{N}_{j}$, we represent $\mathcal{L}$ as the block matrix $\mathcal{L}=$ $\left(\mathcal{L}_{\alpha}^{\beta}\right)_{\alpha, \beta \in l_{N}}$, where $\mathcal{L}_{\alpha}^{\beta}:=\left.\Pi_{\mathbf{H}_{\alpha}} \mathcal{L}\right|_{\mathbf{H}_{\beta}}$. So we can rewrite

$$
\mathcal{L}=\mathcal{D}+\mathcal{T},
$$

where $\mathcal{D}:=\operatorname{diag}_{\alpha \in l_{N}}\left(\mathcal{L}_{\alpha}\right), \mathcal{L}_{\alpha}:=\mathcal{L}_{\alpha}^{\alpha}, \mathcal{T}:=\left(\mathcal{L}_{\alpha}^{\beta}\right)_{\alpha \neq \beta}$.

We define a diagonal matrix corresponding to the matrix $\mathcal{D}$ as $\bar{D}:=\operatorname{diag}_{\alpha \in l_{N}}\left(\bar{L}_{\alpha}\right)$, where $\bar{L}_{\alpha}=$ $\operatorname{diag}_{j \in \Omega_{\alpha}}\left(D_{j}\right)$.

To show $\mathcal{D}$ is invertible, we only need to prove that $\mathcal{L}_{\alpha}$ is invertible, $\forall \alpha \in l_{N}$.

Lemma 2.5. $\forall \alpha \in l_{N}, \mathcal{L}_{\alpha}$ is invertible and $\left\|\mathcal{L}_{\alpha}^{-1}\right\|_{0} \leq C y_{1}^{-1} M_{\alpha}^{\kappa}$.

The proof process of above Lemma is similar with Lemma 6.6 in [4], so we omit it. 
Lemma 2.6. Assume that a is diophantine. We have

$$
\left\|\mathcal{D}^{-1} \bar{D} h\right\|_{s_{1}} \leq c\left(\varsigma, s_{1}, y_{1}\right) N^{\tau}\|h\|_{s_{2}},
$$

where $c\left(\varsigma, s_{1}, y_{1}\right)$ is a constant which depends on $\varsigma, s_{1}$ and $y_{1}$.

Proof. Note that $\left\|h_{\alpha}\right\|_{0} \leq m_{\alpha}^{-s_{1}}\left\|h_{\alpha}\right\|_{s_{1}}$ and $M_{\alpha}=2 m_{\alpha}$. So for any $h=\sum_{\alpha \in l_{N}} h_{\alpha} \in \mathbf{H}_{\alpha}, h_{\alpha} \in \mathbf{H}_{\alpha}$,

$$
\begin{aligned}
\left\|\mathcal{D}^{-1} \bar{D} h\right\|_{s_{1}}^{2} & =\sum_{\alpha \in l_{N}}\left\|\mathcal{L}_{\alpha}^{-1} \bar{L}_{\alpha} h_{\alpha}\right\|_{s_{1}}^{2} \leq \sum_{\alpha \in l_{N}} M_{\alpha}^{2 s_{1}}\left\|\mathcal{L}_{\alpha}^{-1} \bar{L}_{\alpha} h_{\alpha}\right\|_{0}^{2} \\
& \leq c y_{1}^{-2} \sum_{\alpha \in l_{N}} M_{\alpha}^{2\left(s_{1}+\tau\right)}\left\|\bar{L}_{\alpha} h_{\alpha}\right\|_{0}^{2} \\
& \leq c y_{1}^{-2} \sum_{\alpha \in l_{N}} M_{\alpha}^{2\left(s_{1}+\tau\right)} m_{\alpha}^{-2 s_{1}}\left\|\bar{L}_{\alpha} h_{\alpha}\right\|_{s_{1}}^{2} \\
& \leq c y_{1}^{-2} 4^{s_{1}} \sum_{\alpha \in l_{N}} M_{\alpha}^{2 \tau}\left\|\bar{L}_{\alpha} h_{\alpha}\right\|_{s_{1}}^{2} \\
& \leq c y_{1}^{-2} 4^{s_{1}} N^{2 \tau} \sum_{\alpha \in l_{N}}\left\|\bar{L}_{\alpha} h_{\alpha}\right\|_{s_{1}}^{2} \\
& =c y_{1}^{-2} 4^{s_{1}} N^{2 \tau}\|\bar{D} h\|_{s_{1}}^{2} .
\end{aligned}
$$

Using interpolation and (2.8), for $0<s_{1}<s_{2}$, it follows from (2.20) that

$$
\begin{aligned}
\left\|\mathcal{D}^{-1} \bar{D} h\right\|_{s_{1}} & \leq c y_{1}^{-1} 2^{s_{1}} N^{\tau}\|\bar{D} h\|_{s_{1}} \\
& \leq c y_{1}^{-1} 2^{s_{1}} N^{\tau}\left(|\bar{D}|_{s_{2}}\|h\|_{s_{1}}+|\bar{D}|_{s_{1}}\|h\|_{s_{2}}\right) \\
& \leq c(\varsigma) y_{1}^{-1} 2^{s_{1}+1} N^{\tau}\|h\|_{s_{2}} .
\end{aligned}
$$

This completes the proof.

The following result is taken from [4], so we omit the proof.

Lemma 2.7. $\forall s \geq 0, \forall m \in \boldsymbol{N}$, there hold:

$$
\begin{aligned}
& c\left(s_{1}\right)\left\|\mathcal{D}^{-1} \mathcal{T}\right\|_{s_{0}}<\frac{1}{2},\left\|\mathcal{D}^{-1}\right\|_{s} \leq c(s) y_{1}^{-1} N^{\tau}, \\
& \left\|\left(\mathcal{D}^{-1} \mathcal{T}\right)^{m} h\right\|_{s} \leq\left(\varepsilon y^{-1} K(s)\right)^{m}\left(m N^{\kappa_{0}}|T|_{s}|T|_{s_{0}}^{m-1}\|h\|_{s_{0}}+|T|_{s_{0}}^{m}\|h\|_{s}\right) .
\end{aligned}
$$

Lemma 2.8. Assume that $a$ is diophantine. For $0<s_{0}<s_{1}<s_{2}<s_{3}<k-1$, we have

$$
\left\|\mathcal{L}^{-1} h\right\|_{s_{1}} \leq c\left(\varsigma, \tau, s_{1}, y_{1}, y\right) N^{\tau+\kappa_{0}}\left(s_{3}-s_{2}\right)^{-\tau}\left(\|h\|_{s_{3}}+\varepsilon|T|_{s_{1}}\|h\|_{s_{2}}\right) .
$$

Proof. The Neumann series

$$
\mathcal{L}^{-1}=\left(I+\mathcal{D}^{-1} \mathcal{T}\right)^{-1} \mathcal{D}^{-1}=\sum_{m \geq 0}(-1)^{m}\left(\mathcal{D}^{-1} \mathcal{T}\right)^{m} \mathcal{D}^{-1}
$$

is totally convergent in operator norm $\|\cdot\|_{s_{0}}$ with $\left\|\mathcal{L}^{-1}\right\|_{s_{0}} \leq c y_{1}^{-1} N^{\tau}$, by using (2.21).

By (2.22) and (2.24), we have

$$
\begin{aligned}
\left\|\mathcal{L}^{-1} h\right\|_{S_{1}} \leq & \left\|\mathcal{D}^{-1} h\right\|_{s_{1}}+\sum_{m \geq 1}\left\|\left(\mathcal{D}^{-1} \mathfrak{T}\right)^{m} \mathcal{D}^{-1} h\right\|_{s_{1}} \\
\leq & \left\|\mathcal{D}^{-1} h\right\|_{s_{1}}+\left\|\mathcal{D}^{-1} h\right\|_{s_{1}} \sum_{m \geq 1}\left(\varepsilon y_{1}^{-1} K(s)|T|_{s_{0}}\right)^{m} \\
& +N^{K_{0}} K\left(s_{1}\right) \varepsilon y_{1}^{-1}|T|_{s_{1}}\left\|\mathcal{D}^{-1} h\right\|_{s_{0}} \sum_{m \geq 1} m\left(K(s) \varepsilon y_{1}^{-1}|T|_{s_{0}}\right)^{m-1} .
\end{aligned}
$$


Using $\sup _{x>0}\left(x^{y} e^{-x}\right)=\left(y e^{-1}\right)^{y}, \forall y \geq 0$, for $0<s_{1}<s_{2}<s_{3}$, it follows from Lemma 2.4 that

$$
\begin{aligned}
\left\|\mathcal{D}^{-1} h\right\|_{S_{1}}^{2} & =\left\|\mathcal{D}^{-1} \bar{D} \bar{D}^{-1} h\right\|_{S_{1}}^{2} \\
& \leq c^{2}\left(\varsigma, s_{1}, y_{1}\right) N^{2 \tau}\left\|\bar{D}^{-1} h\right\|_{s_{2}}^{2} \\
& =c^{2}\left(\varsigma, s_{1}, y_{1}\right) N^{2 \tau} \sum_{j \in S} e^{2|j| s_{2}}\left\|\bar{D}^{-1} h_{j}\right\|_{\mathbf{L}^{2}}^{2} \\
& \leq c^{2}\left(\varsigma, s_{1}, y_{1}\right) N^{2 \tau} \sum_{j \in S} e^{2|j| s_{2}}\left|\omega_{j}^{2}+\mu-\varepsilon a \omega_{j}^{2 q}\right|^{-2}\left\|h_{j}\right\|_{\mathbf{L}^{2}}^{2} \\
& \leq c^{2}\left(\varsigma, s_{1}, y_{1}\right) N^{2 \tau} \sum_{j \in S} e^{-2|j|\left(s_{3}-s_{2}\right)}|j|^{-2} e^{2|j| s_{3}}\left\|h_{j}\right\|_{\mathbf{L}^{2}}^{2} \\
& \leq c^{2}\left(\varsigma, \tau, s_{1}, y_{1}, y\right) N^{2 \tau}\left(s_{3}-s_{2}\right)^{-2 \tau}\|h\|_{S_{3}}^{2} .
\end{aligned}
$$

Thus by (2.25) and (2.26), we derive

$$
\begin{aligned}
\left\|\mathcal{L}^{-1} h\right\|_{s_{1}} & \leq y_{1}^{-1} N^{\kappa_{0}} K^{\prime}\left(s_{1}\right)\left(\left\|\mathcal{D}^{-1} h\right\|_{s_{1}}+\varepsilon|T|_{s_{1}}\left\|\mathcal{D}^{-1} h\right\|_{s_{0}}\right) \\
& \leq c\left(\varsigma, \tau, s_{1}, y_{1}, y\right) N^{\tau+\kappa_{0}}\left(s_{3}-s_{2}\right)^{-\tau}\left(\|h\|_{s_{3}}+\varepsilon|T|_{s_{1}}\|h\|_{s_{2}}\right),
\end{aligned}
$$

where $0<s_{1}<s_{2}<s_{3}$ and $\varepsilon y_{1}^{-1} \varsigma^{-1}\left(1+|T|_{s_{0}}\right) \leq c(k)$ small enough.

Now we are ready to prove Proposition 2.1. Let

$$
h=h_{R}+h_{S},
$$

where $h_{S} \in \mathbf{H}_{S}, h_{R} \in \mathbf{H}_{R}$. Then by the resolvent identity (2.15),

$$
\begin{aligned}
& \left\|\left(L^{(N)}\right)^{-1} h\right\|_{s_{1}} \\
\leq & \left\|L_{R}^{-1} h_{R}+L_{R}^{-1} L_{S}^{R} \mathcal{L}^{-1}\left(h_{S}+L_{R}^{S} L_{R}^{-1} h_{R}\right)\right\|_{s_{1}}+\left\|\mathcal{L}^{-1}\left(h_{R}+L_{R}^{S} L_{R}^{-1} h_{R}\right)\right\|_{s_{1}} \\
\leq & \left\|L_{R}^{-1} h_{R}\right\|_{s_{1}}+\left\|L_{R}^{-1} L_{S}^{R} \mathcal{L}^{-1} h_{S}\right\|_{s_{1}}+\left\|L_{R}^{-1} L_{S}^{R} \mathcal{L}^{-1} L_{R}^{S} L_{R}^{-1} h_{R}\right\|_{s_{1}} \\
& +\left\|\mathcal{L}^{-1} h_{R}\right\|_{s_{1}}+\left\|\mathcal{L}^{-1} L_{R}^{S} L_{R}^{-1} h_{R}\right\|_{s_{1}} .
\end{aligned}
$$

Next we estimate the right hand side of (2.28) one by one. Using (2.12), (2.17) and (2.23), for $0<s_{1}<s_{2}<s_{3}<$ $s_{4}<k-1$, we have

$$
\begin{aligned}
& \left\|L_{R}^{-1} L_{S}^{R} \mathcal{L}^{-1} h_{S}\right\|_{s_{1}} \\
\leq & c\left(y, \tau, s_{2}\right)\left(s_{2}-s_{1}\right)^{-\tau}\left(1+\varepsilon \varsigma^{-1}|T|_{s_{2}}\right)\left\|L_{S}^{R} \mathcal{L}^{-1} h_{S}\right\|_{s_{2}} \\
\leq & c\left(y, \tau, s_{2}\right)\left(s_{2}-s_{1}\right)^{-\tau}\left(1+\varepsilon \varsigma^{-1}|T|_{s_{2}}\right)|T|_{s_{2}}\left\|\mathcal{L}^{-1} h\right\|_{s_{2}} \\
\leq & c\left(y, y_{1}, \varsigma, \tau, s_{2}\right)\left(s_{2}-s_{1}\right)^{-\tau}\left(s_{4}-s_{3}\right)^{-\tau} N^{\tau+\kappa_{0}} \\
& \times\left(1+\varepsilon \varsigma^{-1}|T|_{s_{2}}\right)|T|_{s_{2}}\left(\|h\|_{s_{3}}+\varepsilon|T|_{s_{2}}\|h\|_{s_{4}}\right),
\end{aligned}
$$

$$
\begin{aligned}
& \left\|\mathcal{L}^{-1} L_{R}^{S} L_{R}^{-1} h_{R}\right\|_{s_{1}} \\
\leq \quad & c\left(\varsigma, \tau, s_{1}, y_{1}, y\right) N^{\tau+\kappa_{0}}\left(s_{3}-s_{2}\right)^{-\tau}\left(\left\|L_{R}^{S} L_{R}^{-1} h_{R}\right\|_{s_{3}}+\varepsilon|T|_{s_{1}}\left\|L_{R}^{S} L_{R}^{-1} h_{R}\right\|_{s_{2}}\right) \\
\leq & c\left(\varsigma, \tau, s_{1}, s_{2}, s_{3}, y_{1}, y\right) N^{\tau+\kappa_{0}}\left(s_{3}-s_{2}\right)^{-\tau} \\
& \times\left(|T|_{s_{3}}\left\|L_{R}^{-1} h_{R}\right\| s_{3}+\varepsilon|T|_{s_{1}}|T|_{s_{2}}\left\|L_{R}^{-1} h_{R}\right\| s_{s_{2}}\right) \\
\leq \quad & c\left(\varsigma, \tau, s_{1}, s_{2}, s_{3}, y_{1}, y\right) N^{\tau+\kappa_{0}}\left(s_{3}-s_{2}\right)^{-\tau}\left(|T|_{s_{3}}\left(s_{4}-s_{3}\right)^{-\tau}\left(1+\varepsilon \varsigma^{-1}|T|_{s_{4}}\right)\|h\|_{S_{4}}\right. \\
& \left.+\varepsilon|T|_{s_{1}}|T|_{s_{2}}\left(s_{3}-s_{2}\right)^{-\tau}\left(1+\varepsilon \varsigma^{-1}|T|_{s_{3}}\right)\|h\|_{s_{3}}\right) \\
\leq \quad & c\left(\varsigma, \tau, s_{1}, s_{2}, s_{3}, y_{1}, y\right) N^{\tau+\kappa_{0}}\left(s_{3}-s_{2}\right)^{-\tau}|T|_{s_{3}}\left(1+\varepsilon \varsigma^{-1}|T|_{s_{4}}\right) \\
& \times\left(\left(s_{4}-s_{3}\right)^{-\tau}\|h\|_{s_{4}}+\varepsilon|T|_{s_{2}}\left(s_{3}-s_{2}\right)^{-\tau}\|h\|_{s_{3}}\right), \\
& \quad\left\|L_{R}^{-1} L_{S}^{R} \mathcal{L}^{-1} L_{R}^{S} L_{R}^{-1} h_{R}\right\|_{s_{1}}
\end{aligned}
$$




$$
\begin{aligned}
\leq & c\left(y, \tau, s_{2}\right)\left(s_{2}-s_{1}\right)^{-\tau}\left(1+\varepsilon \varsigma^{-1}|T|_{s_{2}}\right)\left\|L_{S}^{R} \mathcal{L}^{-1} L_{R}^{S} L_{R}^{-1} h_{R}\right\|_{s_{2}} \\
\leq & c\left(y, \tau, s_{2}\right)\left(s_{2}-s_{1}\right)^{-\tau}\left(1+\varepsilon \varsigma^{-1}|T|_{s_{2}}\right)|T|_{s_{2}}\left\|\mathcal{L}^{-1} L_{R}^{S} L_{R}^{-1} h_{R}\right\|_{s_{2}} \\
\leq & c\left(\varsigma, \tau, s_{1}, s_{2}, s_{3}, y_{1}, y\right) N^{\tau+\kappa_{0}}\left(s_{3}-s_{2}\right)^{-\tau}\left(s_{2}-s_{1}\right)^{-\tau}|T|_{s_{3}}^{2} \\
& \times\left(1+\varepsilon \varsigma^{-1}|T|_{s_{4}}\right)^{2}\left(\left(s_{4}-s_{3}\right)^{-\tau}\|h\|_{s_{4}}+\varepsilon|T|_{s_{2}}\left(s_{3}-s_{2}\right)^{-\tau}\|h\|_{s_{3}}\right) .
\end{aligned}
$$

The terms $\left\|L_{R}^{-1} h_{R}\right\|_{s_{1}}$ and $\left\|\mathcal{L}^{-1} h_{R}\right\|_{s_{1}}$ can be controlled by using (2.17) and (2.23). Thus by (2.28)-(2.31), for $0<s<\tilde{s}$, we conclude

$$
\left\|\left(L^{(N)}\right)^{-1} h\right\|_{s} \leq c\left(\varsigma, \tau, s, \tilde{s}, y_{1}, y\right) N^{\tau+\kappa_{0}}\left(1+\varepsilon \varsigma^{-1}|T|_{\tilde{s}}\right)^{3}(\tilde{s}-s)^{-\tau}\|h\|_{\tilde{s}},
$$

which together with Lemma 2.8 gives (2.7).

\section{Nash-Moser-type iteration scheme}

We define the finite dimensional subspaces

$$
\begin{gathered}
\mathbf{H}_{s}^{\left(N_{i}\right)}=\left\{u \in \mathbf{H}_{s} \mid u=\sum_{|j| \leq N_{i}} u_{j} e^{i j \cdot x}\right\}, \\
\mathbf{H}_{s}^{\left(N_{i}\right) \perp}=\left\{u \in \mathbf{H}_{s} \mid u=\sum_{|j|>N_{i}} u_{j} e^{i j \cdot x}\right\} .
\end{gathered}
$$

Then we have the orthogonal splitting

$$
\mathbf{H}_{s}=\mathbf{H}_{s}^{\left(N_{i}\right)} \bigoplus \mathbf{H}_{s}^{\left(N_{i}\right) \perp},
$$

where $i$ denotes the " $i$ " th iterative step and $\forall s \leq k$. For a given suitable $N_{0}>1$, we take $N_{i} \leq N_{i+1}$ and and $N_{i}=N_{0}^{i}, \forall i \in \mathbf{N}$.

The orthogonal projectors onto $\mathbf{H}_{s}^{\left(N_{i}\right)}$ and $\mathbf{H}_{s}^{\left(N_{i}\right) \perp}$ denote by $\Pi^{\left(N_{i}\right)}: \mathbf{H}_{s} \longrightarrow \mathbf{H}_{s}^{\left(N_{i}\right)}$ and $\Pi^{\left(N_{i}\right) \perp}: \mathbf{H}_{s} \longrightarrow \mathbf{H}_{s}^{\left(N_{i}\right) \perp}$, which satisfy the "smoothing" properties:

$$
\begin{aligned}
& \left\|\Pi^{\left(N_{i}\right)} u\right\|_{s+d} \leq e^{N_{i}^{d}}\|u\|_{s}, \quad \forall u \in X_{s}, \quad \forall s, d \geq 0, \\
& \left\|\Pi^{\left(N_{i}\right) \perp} u\right\|_{s} \leq N_{i}^{-d}\|u\|_{s+d}, \quad \forall u \in X_{s+d}, \quad \forall s, d \geq 0 .
\end{aligned}
$$

Consider

$$
L_{a} u=\varepsilon f(x, u),
$$

where

$$
L_{a}:=-\triangle-\mu+\varepsilon a \triangle^{q} .
$$

The linearized operator of (3.2) has the following form

$$
L_{a}^{\left(N_{i}\right)}:=\left.\Pi^{\left(N_{i}\right)}\left(L_{a}-\varepsilon D_{u} f(\delta, u)\right)\right|_{\mathbf{H}_{s}^{\left(N_{i}\right)}},
$$

where $D$ denotes the Frechet derivative.

By (3.2), we define

$$
\mathcal{J}(u)=L_{a} u-\varepsilon \Pi^{\left(N_{i}\right)} f(x, u)=0 .
$$

Next we construct the first step approximation.

Lemma 3.1. Assume that $a$ is diophantine. Then system (3.4) has the first step approximation $u_{1} \in \boldsymbol{H}_{s}^{\left(N_{1}\right)}$

$$
u_{1}=-\left(L_{a}^{\left(N_{1}\right)}\right)^{-1} E_{0} \in \boldsymbol{H}_{s}^{\left(N_{1}\right)},
$$

and the error term is

$$
E_{1}=R_{0}=-\varepsilon \Pi^{\left(N_{1}\right)}\left(f\left(x, u_{0}+u_{1}\right)-f\left(x, u_{0}\right)-D_{u} f\left(x, u_{0}\right) u_{1}\right) .
$$


Proof. Assume that the 0 th step approximation solution $u_{0}$ satisfies

$$
f\left(x, u_{0}\right) \neq 0 \text {. }
$$

Then the target is to get the 1 th step approximation solution.

Denote

$$
E_{0}=L_{a} u_{0}-\varepsilon \Pi^{\left(N_{1}\right)} f\left(x, u_{0}\right)
$$

By (3.4), we have

$$
\begin{aligned}
\mathcal{J}\left(u_{0}+u_{1}\right)= & L_{a}\left(u_{0}+u_{1}\right)-\varepsilon \Pi^{\left(N_{1}\right)} f\left(x, u_{0}+u_{1}\right) \\
= & L_{a} u_{0}-\varepsilon \Pi^{\left(N_{1}\right)} f\left(x, u_{0}\right)+L_{a} u_{1}+\varepsilon \Pi^{\left(N_{1}\right)} D_{u} f\left(x, u_{0}\right) u_{1} \\
& -\varepsilon \Pi^{\left(N_{1}\right)}\left(f\left(x, u_{0}+u_{1}\right)-f\left(x, u_{0}\right)-D_{u} f\left(x, u_{0}\right) u_{1}\right) \\
= & E_{0}+L_{a}^{\left(N_{1}\right)} u_{1}+R_{0} .
\end{aligned}
$$

Then taking

$$
E_{0}+L_{a}^{\left(N_{1}\right)} u_{1}=0
$$

yields

$$
u_{1}=-\left(L_{a}^{\left(N_{1}\right)}\right)^{-1} E_{0} \in \mathbf{H}_{s}^{\left(N_{1}\right)} .
$$

By (3.8), we denote

$$
\begin{aligned}
E_{1} & :=R_{0}=J_{1}\left(u_{0}+u_{1}\right) \\
& =-\varepsilon \Pi^{\left(N_{1}\right)}\left(f\left(x, u_{0}+u_{1}\right)-f\left(x, u_{0}\right)-D_{u} f\left(x, u_{0}\right) u_{1}\right) .
\end{aligned}
$$

On the other hand, by (3.4) and (3.7), we can obtain

$$
E_{0}=-\varepsilon\left(I-\Pi^{\left(N_{0}\right)}\right) \Pi^{\left(N_{1}\right)} f\left(x, u_{0}\right) .
$$

This completes the proof.

In order to prove the convergence of the Nash-Moser iteration scheme, the following estimate is needed. For convenience, we define

$$
\tilde{E}_{0}:=-\varepsilon \Pi^{\left(N_{1}\right)} f\left(x, u_{0}\right) .
$$

Lemma 3.2. Assume that $a$ is diophantine. Then for any $0<\alpha<\sigma$, the following estimates hold:

$$
\begin{gathered}
\left\|u_{1}\right\|_{\sigma-\alpha} \leq C(\alpha)\left(1+\varepsilon \varsigma^{-1}\left\|u_{0}\right\|_{\sigma}^{p}\right)^{3}\left\|\tilde{E}_{0}\right\|_{\sigma+\tau+\kappa_{0}}, \\
\left\|E_{1}\right\|_{\sigma-\alpha} \leq C^{p}(\alpha)\left(1+\varepsilon \varsigma^{-1}\left\|u_{0}\right\|_{\sigma}^{p}\right)^{3 p}\left\|\tilde{E}_{0}\right\|_{\sigma+\tau+\kappa_{0}}^{p},
\end{gathered}
$$

where $C(\alpha)$ is defined in (3.12).

Proof. Denote

$$
C(\alpha)=c\left(\varsigma, \tau, s, \tilde{s}, y_{1}, y\right) \alpha^{-\tau} \text {. }
$$

From the definition of $u_{1}$ in (3.5), by (2.7), (3.1) and (3.10), we derive

$$
\begin{aligned}
\left\|u_{1}\right\|_{\sigma-\alpha} & =\left\|-\left(L_{a}^{\left(N_{1}\right)}\right)^{-1} E_{0}\right\|_{\sigma-\alpha} \\
& \leq C(\alpha) N_{1}^{\tau+\kappa_{0}}\left(1+\varepsilon \varsigma^{-1}\left\|u_{0}\right\|_{\sigma}^{p}\right)^{3}\left\|E_{0}\right\|_{\sigma} \\
& \leq C(\alpha)\left(1+\varepsilon \varsigma^{-1}\left\|u_{0}\right\|_{\sigma}^{p}\right)^{3}\left\|\tilde{E}_{0}\right\|_{\sigma+\tau+\kappa_{0}} .
\end{aligned}
$$

By assumption (1.4) and the definition of $E_{1}$, we have

$$
\begin{aligned}
\left\|E_{1}\right\|_{\sigma-\alpha} & =\left\|\Pi^{\left(N_{1}\right)}\left(f\left(x, u_{0}+u_{1}\right)-f\left(x, u_{0}\right)-D_{u} f\left(x, u_{0}\right) u_{1}\right)\right\|_{\sigma-\alpha} \\
& \leq\left\|u_{1}\right\|_{\sigma-\alpha}^{p} \\
& \leq C^{p}(\alpha)\left(1+\varepsilon \varsigma^{-1}\left\|u_{0}\right\|_{\sigma}^{p}\right)^{3 p}\left\|\tilde{E}_{0}\right\|_{\sigma+\tau+\kappa_{0}}^{p} .
\end{aligned}
$$

This completes the proof. 
For $i \in \mathbf{N}$ and $0<\sigma_{0}<\bar{\sigma}<\sigma<k-1$, set

$$
\begin{aligned}
& \sigma_{i}:=\bar{\sigma}+\frac{\sigma-\bar{\sigma}}{2^{i}}, \\
& \alpha_{i+1}:=\sigma_{i}-\sigma_{i+1}=\frac{\sigma-\bar{\sigma}}{2^{i+1}}
\end{aligned}
$$

By (3.14)-(3.15), it follows that

$$
\sigma_{0}>\sigma_{1}>\ldots>\sigma_{i}>\sigma_{i+1}>\ldots, \text { for } i \in \mathbf{N}
$$

Define

$$
\begin{gathered}
\mathcal{P}_{1}\left(u_{0}\right):=u_{0}+u_{1}, \text { for } u_{0} \in \mathbf{H}_{\sigma_{0}}^{\left(N_{0}\right)}, \\
E_{i}=\mathcal{J}\left(\sum_{k=0}^{i} u_{k}\right)=\mathcal{J}\left(\mathcal{P}_{1}^{i}\left(u_{0}\right)\right),
\end{gathered}
$$

In fact, to obtain the " $i$ th" approximation solution $u_{i} \in \mathbf{H}_{\sigma_{i}}^{\left(N_{i}\right)}$ of system (3.4), we need to solve following equations

$$
\begin{aligned}
\mathcal{\partial}\left(\sum_{k=0}^{i} u_{k}\right)= & L_{a}\left(\sum_{k=0}^{i-1} u_{k}\right)-\varepsilon \Pi^{\left(N_{i}\right)} f\left(x, \sum_{k=0}^{i-1} u_{k}\right)+L_{a} u_{i}-\varepsilon \Pi^{\left(N_{i}\right)} D_{u} f\left(x, \sum_{k=0}^{i-1} u_{k}\right) u_{i} \\
& -\varepsilon \Pi^{\left(N_{i}\right)}\left(f\left(x, \sum_{k=0}^{i} u_{k}\right)-f\left(x, \sum_{k=0}^{i-1} u_{k}\right)-D_{u} f\left(x, \sum_{k=0}^{i-1} u_{k}\right) u_{i}\right) .
\end{aligned}
$$

Then, we get the ' $i$ th' step approximation $u_{i} \in \mathbf{H}_{\sigma_{i}}^{\left(N_{i}\right)}$ :

$$
u_{i}=-\left(L_{a}^{\left(N_{i}\right)}\right)^{-1} E_{i-1},
$$

where

$$
E_{i-1}=L_{a}\left(\sum_{k=0}^{i-1} u_{k}\right)-\varepsilon \Pi^{\left(N_{i}\right)} f\left(x, \sum_{k=0}^{i-1} u_{k}\right)=-\varepsilon\left(I-\Pi^{\left(N_{i-1}\right)}\right) \Pi^{\left(N_{i}\right)} f\left(x, \sum_{k=0}^{i-1} u_{k}\right) .
$$

As done in Lemma 3.2, it is easy to get that

$$
\begin{gathered}
E_{i}:=R_{i-1}=-\varepsilon \Pi^{\left(N_{i}\right)}\left(f\left(x, \sum_{k=0}^{i-1} u_{k}\right)-f\left(x, \sum_{k=0}^{i} u_{k}\right)-D_{u} f\left(x, \sum_{k=0}^{i-1} u_{k}\right) u_{i}\right), \\
\tilde{E}_{i}=-\varepsilon \Pi^{\left(N_{i}\right)} f\left(x, \sum_{k=0}^{i-1} u_{k}\right) .
\end{gathered}
$$

Hence, we only need to estimate $R_{i-1}$ to prove the convergence of algorithm. In the following, a sufficient condition on the convergence of the Nash-Moser iteration scheme is proved. This proof is based on Lemma 3.2. It also shows the existence of solutions for (3.4).

Lemma 3.3. Assume that $a$ is diophantine. Then for sufficiently small $\varepsilon$, equations (3.2) has a solution

$$
u_{\infty}=\sum_{k=0}^{\infty} u_{k} \in \boldsymbol{H}_{\bar{\sigma}} \cap \mathcal{B}_{1}(0),
$$

where $\mathcal{B}_{1}(0):=\left\{u \mid\|u\|_{s} \leq 1, \forall s>\bar{s}>0\right\}$

Proof. We divide into two cases. If $\varepsilon \varsigma^{-1}\left\|u_{i-1}\right\|_{\sigma_{i-1}}^{p}<1$, by (2.7), (3.16) and (3.18), we derive

$$
\begin{aligned}
\left\|u_{i}\right\|_{\sigma_{i}} & =\left\|-\left(L_{a}^{\left(N_{i}\right)}\right)^{-1} E_{i-1}\right\|_{\sigma_{i}} \\
& \leq C\left(\alpha_{i}\right) N_{i}^{\tau+\kappa_{0}}\left(1+\varepsilon \varsigma^{-1}\left\|u_{i-1}\right\|_{\sigma_{i-1}}^{p}\right)^{3}\left\|E_{i-1}\right\| \sigma_{i-1}
\end{aligned}
$$




$$
\begin{aligned}
& \leq C\left(\alpha_{i}\right)\left(1+\varepsilon \varsigma^{-1}\left\|u_{i-1}\right\|_{\sigma_{i-1}}^{p}\right)^{3}\left\|\tilde{E}_{i-1}\right\| \sigma_{i-1}+\tau+\kappa_{0} \\
& \leq \quad 2 C\left(\alpha_{i}\right)\left\|\tilde{E}_{i-1}\right\|_{\sigma_{i-1}+\tau+\kappa_{0}},
\end{aligned}
$$

where $c(\varepsilon, \varsigma)$ is a constant depending on $\varepsilon$ and $\varsigma$.

Note that $N_{i}=N_{0}^{i}, \forall i \in \mathbf{N}$. By (3.17)-(3.19) and assumption (1.4), we have

$$
\begin{aligned}
\left\|E_{i}\right\|_{\sigma_{i}} & =\varepsilon\left\|\Pi^{\left(N_{i}\right)}\left(f\left(x, \sum_{k=0}^{i} u_{k}\right)-f\left(x, \sum_{k=0}^{i-1} u_{k}\right)-D_{u} f\left(x, \sum_{k=0}^{i-1} u_{k}\right) u_{i}\right)\right\|_{\sigma_{i}} \\
& \leq \varepsilon c(s)\left\|u_{i}\right\|_{\sigma_{i}}^{p} \\
& \leq \varepsilon c(s) N_{i}^{\left(\tau+\kappa_{0}\right) p} C^{p}\left(\alpha_{i}\right)\left\|E_{i-1}\right\|_{\sigma_{i-1}}^{p} \\
& \leq(\varepsilon c(s))^{p+1} N_{i}^{\left(\tau+\kappa_{0}\right) p} N_{i-1}^{\left(\tau+\kappa_{0}\right) p^{2}} C^{p}\left(\alpha_{i}\right) C^{p^{2}}\left(\alpha_{i-1}\right)\left\|E_{i-2}\right\|_{\sigma_{i-2}}^{p^{2}} \\
& \leq \cdots \\
& \leq(\varepsilon c(s))^{\sum_{k=1}^{i-1} p^{k}+1} N_{0}^{\left(\tau+\kappa_{0}\right) p^{i+2}}\left\|E_{0}\right\|_{\sigma_{0}}^{p^{i}} \prod_{k=1}^{i} C^{p^{k}}\left(\alpha_{i+1-k}\right) \\
& \leq(\varepsilon c(s))^{p^{i}}(\varepsilon, \varsigma)\left(N_{0}^{\left(\tau+\kappa_{0}\right) p^{2}}\left\|E_{0}\right\|_{\sigma_{0}}\right)^{p^{i}} \prod_{k=1}^{i} C^{p^{k}}\left(\alpha_{i+1-k}\right) \\
\leq & (\varepsilon c(s))^{p^{i}}(\varepsilon, \varsigma)\left\|\tilde{E}_{0}\right\|_{\sigma_{0}+\left(\tau+\kappa_{0}\right) p^{2}}^{p^{i}} \prod_{k=1}^{i} C^{p^{k}}\left(\alpha_{i+1-k}\right) \\
\leq & \left(8^{p^{2}} \varepsilon c(s) c^{p^{2}}\left(\tau, \sigma, \tilde{\sigma}, y_{1}, y\right)\left\|\tilde{E}_{0}\right\|_{\sigma_{0}+\left(\tau+\kappa_{0}\right) p^{2}}\right)^{p^{i}} .
\end{aligned}
$$

Hence, choosing small $\varepsilon>0$ such that

$$
8^{p^{2}} \varepsilon c(s) c^{p^{2}}\left(\tau, \sigma, \tilde{\sigma}, y_{1}, y\right)\left\|\tilde{E}_{0}\right\|_{\sigma_{0}+\left(\tau+\kappa_{0}\right) p^{2}}=8^{p^{2}} \varepsilon c(s) c^{p^{2}}\left(\tau, \sigma, \tilde{\sigma}, y_{1}, y\right) N_{0}^{\left(\tau+\kappa_{0}\right) p^{2}}\left\|\tilde{E}_{0}\right\|_{\sigma_{0}}<1 .
$$

For any fixed $p>1$, we derive

$$
\lim _{i \longrightarrow \infty}\left\|E_{i}\right\|_{\sigma_{i}}=0 .
$$

If $\varepsilon \varsigma^{-1}\left\|u_{i-1}\right\|_{\sigma_{i-1}}^{p} \geq 1$, by (2.7), (3.16) and (3.18), we derive

$$
\begin{aligned}
\left\|u_{i}\right\| \sigma_{i} & =\left\|-\left(L_{a}^{\left(N_{i}\right)}\right)^{-1} E_{i-1}\right\| \sigma_{i} \\
& \leq C\left(\alpha_{i}\right) N_{i}^{\tau+\kappa_{0}}\left(1+\varepsilon \varsigma^{-1}\left\|u_{i-1}\right\|_{\sigma_{i-1}}^{p}\right)^{3}\left\|E_{i-1}\right\|_{\sigma_{i-1}} \\
& \leq 2 \varepsilon^{3} \varsigma^{-3} C\left(\alpha_{i}\right)\left\|u_{i-1}\right\|_{\sigma_{i-1}}^{3 p}\left\|\tilde{E}_{i-1}\right\| \sigma_{i-1}+\tau+\kappa_{0} \\
& \leq\left(2 \varepsilon \varsigma^{-1}\right)^{3(p+1)} C\left(\alpha_{i}\right) C^{3 p}\left(\alpha_{i-1}\right)\left\|u_{i-2}\right\|_{\sigma_{i-2}}^{3 p)^{2}}\left\|\tilde{E}_{i-2}\right\|_{\sigma_{i-2}+\tau+\kappa_{0}}^{3 p}\left\|\tilde{E}_{i-1}\right\|_{\sigma_{i-1}+\tau+\kappa_{0}} \\
& \leq \cdots \\
& \leq\left(2 \varepsilon \varsigma^{-1}\right)^{\sum_{k=0}^{i-1}(3 p)^{k}}\left\|u_{0}\right\|_{\sigma_{0}}^{(3 p)^{i}} \prod_{k=1}^{i} C^{(3 p)^{k-1}}\left(\alpha_{i+1-k}\right)\left\|\tilde{E}_{i-k}\right\|_{\sigma_{i-k}+\tau+\kappa_{0}}^{(3 p} .
\end{aligned}
$$

But we will choose the initial step $u_{0}=0$ in this paper, which combining with (3.22) leads to $\left\|u_{i}\right\|_{\sigma_{i}}=0, \forall i \in \mathbf{N}$. This contradicts with assumption $\varepsilon \varsigma^{-1}\left\|u_{i-1}\right\|_{\sigma_{i-1}}^{p}>1$. Hence, the case is not possible. (3.2) has a solution

$$
u_{\infty}:=\sum_{k=0}^{\infty} u_{k} \in \mathbf{H}_{\bar{\sigma}} \cap \mathcal{B}_{1}(0),
$$

where $\mathcal{B}_{1}(0):=\left\{u \mid\|u\|_{s} \leq 1, \forall s>\bar{s}>0\right\}$. This completes the proof.

Next result gives the local uniqueness of solutions for equation (3.2).

Lemma 3.4. Assume that a is diophantine. Equation (3.2) has a unique solution $u \in \boldsymbol{H}_{\bar{\sigma}} \cap \boldsymbol{B}_{1}(0)$ obtained in Lemma 3.3. 
Proof. Let $u, \tilde{u} \in \mathbf{H}_{\bar{\sigma}} \cap \mathbf{B}_{1}$ (0) be two solutions of system (3.4), where

$$
\mathbf{B}_{1}(0):=\left\{u \mid\|u\|_{s}<\delta \text {, for some } \delta<1, \forall s>\sigma_{0}\right\} .
$$

Write $h=u-\tilde{u}$. Our target is to prove $h=0$. By (3.4), we have

$$
L_{a} h-\varepsilon \Pi^{\left(N_{i}\right)} D_{u} f(x, u) h-\varepsilon \Pi^{\left(N_{i}\right)}\left(f(x, u)-f(x, \tilde{u})-D_{u} f(x, u) h=0,\right.
$$

which implies that

$$
h=\varepsilon\left(L_{a}-\varepsilon \Pi^{\left(N_{i}\right)} D_{u} f(x, u)\right)^{-1} \Pi^{\left(N_{i}\right)}\left(f(x, u)-f(x, \tilde{u})-D_{u} f(x, u) h\right) .
$$

Note that $N_{i}=N_{0}^{i}, \forall i \in \mathbf{N}$. Thus, by (2.7) and (3.23), we have

$$
\begin{aligned}
\|h\|_{\sigma_{i}} & =\varepsilon\left\|\left(L_{a}^{N_{i}}\right)^{-1} \Pi^{\left(N_{i}\right)}\left(f(x, u)-f(x, \tilde{u})-D_{u} f(x, u) h\right)\right\|_{\sigma_{i}} \\
& \leq C\left(\alpha_{i}\right) N_{i}^{\tau+\kappa_{0}}\left(1+\varepsilon \varsigma^{-1}\|u\|_{\sigma_{i-1}}^{p}\right)\|h\|_{\sigma_{i-1}}^{p} \\
& \leq 2^{p+1} N_{i}^{\left(\tau+\kappa_{0}\right)} N_{i-1}^{\left(\tau+\kappa_{0}\right) p} C\left(\alpha_{i}\right) C^{p}\left(\alpha_{i-1}\right)\|h\|_{\sigma_{i-2}}^{p} \\
& \leq \cdots \\
& \leq 2^{\sum_{k=0}^{i-1} p^{k}} N_{0}^{\left(\tau+\kappa_{0}\right)\left(\sum_{k=0}^{i-1} p^{k}\right)}\|h\|_{\sigma_{0}}^{p^{i}} \prod_{k=1}^{i} C^{p^{k-1}}\left(\alpha_{i+1-k}\right) \\
& \leq\left(8^{p^{2}} c^{p^{2}}\left(\varepsilon, \varsigma, \tau, s, \tilde{s}, y_{1}, y\right) N_{0}^{\left(\tau+\kappa_{0}\right) p}\|h\|_{\sigma_{0}}\right)^{p^{i}} .
\end{aligned}
$$

Choosing $\delta<8^{-p^{2}} c^{-p^{2}}\left(\varepsilon, \varsigma, \tau, s, \tilde{s}, y_{1}, y\right) N_{0}^{-\left(\tau+\kappa_{0}\right) p}$, we obtain

$$
\lim _{i \longrightarrow \infty}\|h\|_{\bar{\sigma}}=0 \text {. }
$$

This completes the proof.

Remark 3.1. The dependence upon the parameter, as is well known, is more delicated since it involves in the small divisors of $\omega_{j}$ : it is, however, standard to check that this dependence is $\boldsymbol{C}^{1}$ on a bounded set of Diophantine numbers, for more details, see, for example, [3, 4].

By Lemma 3.1, for sufficient small $\delta_{0}>0$ and given $r>0$, we define

$$
\begin{aligned}
& Y_{y_{1}, \kappa_{0}}^{(N)}:=\left\{\left(\delta, q^{\prime}\right) \in\left[0, \delta_{0}\right) \times \mathbf{H}^{(N)} \mid\left\|q^{\prime}\right\|_{\bar{\sigma}} \leq 1, \varepsilon \delta \text { satisfies }(2.5)-(2.6)\right\}, \\
& U_{r}^{(N)}:=\left\{u \in \mathbf{C}^{1}\left(\left[0, \delta_{0}\right), \mathbf{H}^{(N)}\right) \mid\|u\|_{\bar{\sigma}} \leq 1,\left\|\partial_{\delta} u\right\|_{\bar{\sigma}} \leq r\right\}, \\
& \mathcal{G}_{y_{1}, \kappa_{0}}^{(N)}:=\left\{\delta \in\left[0, \delta_{0}\right) \mid(\delta, u(\delta)) \in Y_{y_{1}, \kappa_{0}}^{(N)} \text { and } u \in U_{r}^{(N)}\right\}, \\
& \mathcal{G}_{r}:=\left\{\delta \in\left[0, \delta_{0}\right) \mid\left\|\left(L_{a}^{(r)}\left(\delta, q^{\prime}(\delta)\right)\right)^{-1}\right\| \leq \frac{4 r^{\kappa}}{y_{1}}\right\}, \\
& \mathcal{G}:=\left\{\delta \in\left[0, \delta_{0}\right) \mid \omega(\delta) \text { satisfies }(2.5)\right\} .
\end{aligned}
$$

Then for a given function $\delta \mapsto q^{\prime}(\delta) \in U_{r}^{(N)}$, the set $\mathcal{G}_{y_{1}, \kappa_{0}}^{(N)}$ is equivalent to

$$
\mathcal{G}_{y_{1}, \kappa_{0}}^{(N)}=\cap_{1 \leq r \leq N} \mathcal{G}_{r} \cap \mathcal{G} \text {. }
$$

Choosing $\kappa$ and $y_{1}$ such that

$$
\kappa \geq \max \left\{\tau, 2+d+n+\frac{2 q-2}{2 q-1}(\tau+2 \varrho)\right\}, y_{1} \in\left(0, y_{2}\right], \text { for } y_{2} \leq y_{1} .
$$

Next we have the measure estimate. The proof of it will be given in Appendix.

Lemma 3.5. (Measure estimates) Assume that a is diophantine, $\varepsilon_{0} y^{-1} M^{\tau+2 q}$ is sufficient small and (3.24) holds. Then $\mathcal{G}_{y_{1}, k_{0}}^{(M)}(0)=\mathcal{G}$, and $\mathcal{G}$ satisfies

$$
\left|\left(\mathcal{G}_{y_{1}, \kappa_{0}}^{(M)}(0)\right)^{c} \cap[0, \delta)\right| \leq C y_{1} \delta, \forall \delta \in\left(0, \delta_{0}\right] .
$$


Furthermore, for any $r^{\prime}>0$, there exists $\delta^{\prime}:=\delta^{\prime}\left(y_{1}, r^{\prime}\right)$ such that the measure estimate

$$
\left|\left(\mathcal{G}_{y_{1}, \kappa_{0}}^{\left(N^{\prime}\right)}\left(u_{2}\right)\right)^{c} \backslash\left(\mathcal{G}_{y_{1}, \kappa_{0}}^{(N)}\left(u_{1}\right)\right)^{c} \cap[0, \delta)\right| \leq C y_{1} \delta N^{-1}, \forall \delta \in\left(0, \delta^{\prime}\right]
$$

holds, where $N^{\prime} \geq N \geq M, u_{1} \in U_{r^{\prime}}^{(N)}, u_{1} \in U_{r^{\prime}}^{(N)}$ with $\left\|u_{2}-u_{1}\right\|_{\bar{\sigma}} \leq N^{-e}$, e denotes a constant depending on $\kappa_{0}$ and $n$.

Proof. This proof follows essentially the scheme of [3-5]. Note that $|j| \leq r$ and the eigenvalue of the operator $L_{a}^{(r)}$ has the form $\omega_{j}^{2}+\mu-\varepsilon a \omega_{j}^{2 q}-O(\varepsilon)$ of the operator $L_{a}^{(r)}$. Here $j \in \mathbf{Z}^{n}$. For sufficient small $\varepsilon_{0} y^{-1} M^{\tau+2 q}$, by (1.8), all the eigenvalues of $L_{a}^{(r)}$ has modulus $\geq y\left(4 r^{\tau}\right)^{-1} \geq y_{1}\left(4 r^{\kappa}\right)^{-1}$. Thus $\mathcal{G}_{r}=\left[0, \delta_{0}\right)$ and the measure estimate (3.25) for $\mathcal{G}$ is standard. To prove the measure estimate (3.26), we divide the process of proof into two cases. For the case $N, N^{\prime} \leq N_{\varepsilon_{0}}:=\left(c y_{1} \varepsilon_{0}^{-1}\right)^{\frac{1}{\tau+2}}, \mathcal{G}_{y_{1}, \kappa_{0}}^{\left(N^{\prime}\right)}\left(u_{2}\right)=\mathcal{G}_{y_{1}, \kappa_{0}}^{(N)}\left(u_{1}\right)=\mathcal{G}$, by the same process of proof of (3.25), one can prove (3.26) holds. For other cases, it is sufficient to prove

$$
\left|\left(\mathcal{G}_{y_{1}, \kappa_{0}}^{\left(N^{\prime}\right)}\left(u_{2}\right)\right)^{c} \backslash\left(\mathcal{G}_{y_{1}, \kappa_{0}}^{(N)}\left(u_{1}\right)\right)^{c} \cap\left[\frac{\delta_{1}}{2}, \delta_{1}\right)\right| \leq C y_{1} \delta N^{-1}, \forall \delta_{1} \in\left[0, \delta_{0}\right] .
$$

For fixed $\delta_{1}$ and the decomposition $\left[0, \delta_{0}\right]=\cup_{n \geq 1}\left[\delta_{0} 2^{-n}, \delta_{0} 2^{-(n-1)}\right]$, we consider the complementary sets in $\left[\frac{\delta_{1}}{2}, \delta_{1}\right)$

$$
\begin{aligned}
\left(\mathcal{G}_{y_{1}, \kappa_{0}}^{\left(N^{\prime}\right)}\left(u_{2}\right)\right)^{c} \backslash\left(\mathcal{G}_{y_{1}, \kappa_{0}}^{(N)}\left(u_{1}\right)\right)^{c} & =\left(\mathcal{G}_{y_{1}, \kappa_{0}}^{\left(N^{\prime}\right)}\left(u_{2}\right)\right)^{c} \cap \mathcal{G}_{y_{1}, \kappa_{0}}^{(N)}\left(u_{1}\right) \\
& \subset\left[\cup_{r \leq N}\left(\mathcal{G}_{r}^{c}\left(u_{2}\right) \cap \mathcal{G}_{r}\left(u_{1}\right) \cap \mathcal{G}\right)\right] \cup\left[\cup_{r>N} \mathcal{G}_{r}^{c}\left(u_{2}\right) \cap \mathcal{G}\right] .
\end{aligned}
$$

If $r \leq N_{\varepsilon_{0}}$, then $\mathcal{G}_{r}^{c}\left(u_{2}\right) \cap \mathcal{G}=\emptyset$. So it is sufficient to prove that, if $\left\|u_{1}-u_{2}\right\|_{\bar{\sigma}} \leq N^{-e}, e \geq d+n+3$, then

$$
\Omega:=\sum_{N_{\varepsilon}<r \leq N}\left|\mathcal{G}_{r}^{c}\left(u_{2}\right) \cap \mathcal{G}_{r}\left(u_{1}\right)\right|+\sum_{r>\max \left\{N, N_{\varepsilon}\right\}}\left|\mathcal{G}_{r}^{c}\left(u_{2}\right)\right| \leq C^{\prime} y_{1} \delta_{1} N^{-1} .
$$

Note that $\left\|\left(L_{a}^{(r)}\right)^{-1}\right\|_{0}$ is the inverse of the eigenvalue of smallest modulus and

$$
\left\|L_{a}^{(r)}\left(u_{2}\right)-L_{a}^{(r)}\left(u_{1}\right)\right\|_{0}=O\left(\varepsilon\left\|u_{2}-u_{1}\right\|_{s_{0}}\right)=O\left(\varepsilon N^{-e}\right) .
$$

The sufficient and necessary condition of an eigenvalues of $L_{a}^{(r)}\left(u_{2}\right)$ in $\left[-4 y_{1} r^{-\tau}-C \varepsilon N^{-e}, 4 y_{1} r^{-\tau}+C \varepsilon N^{-e}\right]$ is that there exists an eigenvalues of $L_{a}^{(r)}\left(u_{1}\right)$ in $\left[-4 y_{1} r^{-\tau}, 4 y_{1} r^{-\tau}\right]$. Thus, it leads to

$$
\begin{aligned}
\mathcal{G}_{r}^{c}\left(u_{2}\right) \cap \mathcal{G}_{r}\left(u_{1}\right) \subset\left\{\delta \in\left[\frac{\delta_{1}}{2}, \delta_{1}\right] \mid \quad\right. & \exists \text { at leat an eigenvalue of } L_{a}^{(r)}\left(\delta, u_{1}\right) \\
& \text { with modulus in } \left.\left[4 y_{1} r^{-\tau}, 4 y_{1} r^{-\tau}+C \varepsilon N^{-e}\right]\right\} .
\end{aligned}
$$

Next we claim that if $\varepsilon$ is small enough and $I$ is a compact interval in $\left[-y_{1}, y_{1}\right]$ of length $|I|$, then

$$
\begin{aligned}
\mid\left\{\delta \in\left[\frac{\delta_{1}}{2}, \delta\right] \text { s.t. at least } \quad\right. & \left.\exists \text { an eigenvalue of } L^{(r)}\left(\delta, u_{1}\right) \text { belongs to } I\right\} \mid \\
& \leq C r^{d+n+1} \delta_{1}^{-(2 q-2)}|I| .
\end{aligned}
$$

Due to the $C^{1}$ map $\delta \mapsto L^{(r)}\left(\delta, u_{1}\right)$ and the selfadjoint property of $L^{(r)}\left(\delta, u_{1}\right)$, we have the corresponding eigenvalue function $\lambda_{k}\left(\delta, u_{1}\right)$ with $1 \leq k \leq r$. Denote the eigenspace of $L^{(r)}\left(\delta, u_{1}\right)$ by $E_{\delta, k}$ associated to $\lambda_{k}\left(\delta, u_{1}\right)$, then by $\left\|\partial_{\delta} b\right\|_{s}=\left\|\left(\partial_{u}^{2} f\right)(x, u)\right\|_{s} \leq C y_{1}^{-1}$ and $\left\|\nabla^{q} h\right\|_{0}^{2} \geq\|h\|_{0}^{2}$, for sufficient small $0<\varepsilon \leq \varepsilon_{0}\left(y_{1}\right)$, we have

$$
\begin{aligned}
\left(\partial_{\delta} \lambda_{k}\left(\delta, u_{1}\right)\right) & \leq \max _{h \in E_{\delta, k},\|h\|_{0}=1}\left(\left(\partial_{\delta} L^{(r)}\right)\left(\delta, u_{1}\right) h, h\right)_{0} \\
& \leq \max _{h \in E_{\delta, k},\|h\|_{0}=1}\left((2 q-1) \delta^{2 q-2}\left(\triangle^{q} h, h\right)_{0}+O\left(\varepsilon y_{1}^{-1}\right)\right) \\
& \leq \max _{h \in E_{\delta, k},\|h\|_{0}=1}\left(-(2 q-1) \delta^{2 q-2}\left\|\nabla^{q} h\right\|_{0}^{2}+O\left(\varepsilon y_{1}^{-1}\right)\right) \\
& \leq \max _{h \in E_{\delta, k},\|h\|_{0}=1}\left(-(2 q-1) \delta^{2 q-2}\|h\|_{0}^{2}+O\left(\varepsilon y_{1}^{-1}\right)\right)
\end{aligned}
$$




$$
\leq-(2 q-1) \delta^{2 q-2}+O\left(\varepsilon y_{1}^{-1}\right) \leq-2\left(q^{\prime}-1\right) \delta_{1}^{2 q-2} .
$$

Hence we have $\left|\lambda_{k}^{-1}\left(I, u_{1}\right) \cap\left[\frac{\delta_{1}}{2}, \delta_{1}\right]\right| \leq C|I| \delta_{1}^{-(2 q-2)}$. The claim holds.

Thus, we obtain

$$
\left|\mathcal{G}_{r}^{c}\left(u_{2}\right) \cap \mathcal{G}_{r}\left(u_{1}\right)\right| \leq C \varepsilon r^{d+n+1} \delta_{1}^{-(2 q-2)} N^{-e} \leq C \delta_{1} N^{-e} r^{d+n+1} .
$$

Furthermore, by (3.27), we have $\left|\mathcal{G}_{r}^{c}\left(u_{2}\right)\right| \leq C y_{1} r^{d+n-\tau+1} \delta_{1}^{-(2 q-2)}$. Therefore, we obtain

$$
\begin{aligned}
\Omega & =\sum_{N_{\varepsilon}<r \leq N}\left|\mathcal{G}_{r}^{C}\left(u_{2}\right) \cap \mathcal{G}_{r}\left(u_{1}\right)\right|+\sum_{r>\max \left\{N, N_{\varepsilon}\right\}}\left|\mathcal{G}_{r}^{C}\left(u_{2}\right)\right| \\
& \leq C \delta_{1}\left(\sum_{r \leq N} r^{d+n+1}\right) N^{-e}+C y_{1} \delta_{1}^{-(2 q-2)} \sum_{r>\max \left\{N, N_{\varepsilon}\right\}} r^{d+n-\tau+1} \\
& \leq C^{\prime}\left(\delta_{1} N^{d+n-e+2}+y_{1} \delta_{1}^{-(2 q-2)}\left(\max \left\{N, N_{\varepsilon}\right\}\right)^{d+n-\tau+2}\right) \\
& \leq C^{\prime \prime} y_{1} \delta_{1} N^{-1},
\end{aligned}
$$

where $C, C^{\prime}$ and $C^{\prime \prime}$ denote constants. This completes the proof.

\section{Acknowledgements}

The first author is supported by Huizhou University Professor Doctor Launch Project Grant, No. 20187 B037. The second author is supported by NSFC No 11771359, and the Fundamental Research Funds for the Central Universities (Grant No. 20720190070, 20720180009 and 201709000061).

\section{References}

[1] A. Bahrouni, H. Ounaies, V.D. Rădulescu, Compactly supported solutions of Schrödinger equations with small perturbation. Appl. Math. Lett. 84 (2018), 148-154.

[2] F.G. Bass, N.N. Nasanov, Nonlinear electromagnetic spin waves. Physics Reports. 189 (1990), 165-223.

[3] M. Berti, M. Bolle, Sobolev periodic solutions of nonlinear wave equations in higher spatial dimensions. Arch. Ration. Mech. Anal. 159 (2010), 609-642.

[4] M. Berti, M. Procesi, Nonlinear wave and Schrödinger equations on compact Lie groups and homogeneous spaces. Duke. Math. J 159 (2011), 479-538.

[5] J. Bourgain, Construction of periodic solutions of nonlinear wave equations in higher dimension. Geom. Funct. Anal. 5 (1995) 629-639.

[6] J. Bourgain, Quasi-periodic solutions of Hamiltonian perturbations of 2D linear Schrödinger equations. Ann. Math. 148 (1998) 363-439.

[7] J. Bourgain, On the Cauchy and invariant measure problem for the periodic Zakharov system. Duck. Math. J 76 (1994) 175202.

[8] S.T. Chen, B.L. Zhang, X.H. Tang, Existence and concentration of semiclassical ground state solutions for the generalized Chern-Simons-Schrödinger system in $H^{1}\left(\mathbb{R}^{2}\right)$. Nonlinear Anal. 185 (2019) 68-96.

[9] F. Gladiali, M. Squassina, Uniqueness of ground states for a class of quasi-linear elliptic equations. Adv. Nonlinear Anal. 1 (2012) 159-179.

[10] Q. Han, J.X. Hong, C.S. Lin, Small divisors in nonlinear elliptic equations. Calc.Var.PDE. 18 (2003) 31-56.

[11] L. Hörmander, Implicit function theorems. Stanford Lecture notes, University, Stanford 1977

[12] C. Ji, F. Fang, Standing waves for the Chern-Simons-Schrödinger equation with critical exponential growth. J. Math. Anal. Appl. 450 (2017) 578-591.

[13] T. Kato, Locally coercive nonlinear equations, with applications to some periodic solutions. Duck. Math. J 51 (1984) 923936.

[14] C.E. Kenig, G. Ponce, L, Vega, The Cauchy problem for quasi-linear Schrödinger equations. Invent. Math. 158 (2004) 343388.

[15] A.M. Kosevich, B.A. Ivanov, A.S. Kovalev, Magnetic solitons. Physics Reports. 194 (1990), 117-238.

[16] S. Kurihura, Large-amplitude quasi-solitons in superfluid films. J. Phys. Soc. Japan. 50 (1981), 3262-3267.

[17] H. Lange, M. Poppenberg, H. Teismann, Nash-Moser methods for the solution of quasilinear schrödinger equations. Comm.PDE. 24 (1999), 1399-1418.

[18] J. Moser, A rapidly converging iteration method and nonlinear partial differential equations I-II. Ann. Scuola Norm. Sup. Pisa. 20, 265-313, 499-535 (1966) 
[19] J. Nash, The embedding for Riemannian manifolds. Amer. Math. 63 (1956) 20-63

[20] P. Korman, On existence of solutions for two classes of nonlinear problems. Comm. PDE. 14 (1989) 519-539.

[21] N.S. Papageorgiou, V.D. Rădulescu, D.D. Repovš, Periodic solutions for a class of evolution inclusions. Comput. Math. Appl. 75 (2018) 3047-3065.

[22] M. Poppenberg, Smooth solutions for a class of fully nonlinear Schrödinger type equations. Nonlinear Anal., Theory Methods Appl. 45, (2001) 723-741

[23] P.L. Sulem, C. Sulem, Nonlinear Schrödinger Equations: Self-Focusing and Wave Collapse. New York: Springer 1999

[24] P. Rabinowitz, A rapid convergence method and a singular perturbation problem. Ann. Inst. Henri Poincaré. 1 (1984) 1-17.

[25] T.F. Xue, C.L. Tang, Existence of a bound state solution for quasilinear Schrödinger equations. Adv. Nonlinear Anal. 8 (2019) 323-338.

[26] W.P. Yan, The motion of closed hypersurfaces in the central force field. J. Diff. Eqns. 261 (2016), 1973-2005.

[27] X. Zhang, B.L. Zhang, M.Q. Xiang, Ground states for fractional Schrödinger equations involving a critical nonlinearity. Adv. Nonlinear Anal. 5 (2016) 293-314. 